Supplemental Material to:

\title{
Spontaneous formation of KCl aggregates in biomolecular simulations: a force field issue?
}

\author{
Pascal Auffinger*, Thomas E. Cheatham III $^{1}$ and Andrea C. Vaiana ${ }^{2}$ \\ Architecture et réactivité de l'ARN, \\ Université Louis Pasteur de Strasbourg, CNRS, IBMC, \\ 15 rue René Descartes, \\ 67084 Strasbourg, \\ France
}

\section{Computational methods section related to Figure 1}

A series of $>40$ ns simulations were performed on d(CGCGAATTCGCG $)_{2}$ duplexes initially in a canonical A-DNA geometry in $4.0 \mathrm{M}$ aqueous $\mathrm{NaCl}$ solution. The equilibrated box size was $\sim 42 \AA$ on a side in a truncated octahedral geometry. In addition to the DNA, net-neutralizing $\mathrm{Na}^{+}$ cations were added along with an extra $142 \mathrm{NaCl}$ pairs. The unit cell contained 1455 waters and was build with both the AMBER ff94 and ff99 force fields. Transition to a B-DNA (essentially infinite B-DNA helix) occurred during the first $5 \mathrm{~ns}$ of simulation. Simulation protocols are described in more detail in (Cheatham, T. E., and M. A. Young. 2000. Molecular dynamics simulation of nucleic acids: successes, limitations, and promise. Biopolymers 56:232-256).

\section{Movie Legends:}

Movie S1: Movie showing $5 \mathrm{~ns}$ of MD trajectories for the Dang_SPC/E_1.0M (no aggregation; left) and Amber_TIP3P_1.OM (aggregation; right) simulations. The $\mathrm{K}^{+}$and $\mathrm{Cl}^{-}$ions are shown in green and cyan, respectively. For clarity, water molecules are not shown.

Movie S2: $\quad$ Movie showing $10 \mathrm{~ns}$ of MD trajectories for the Dang_SPC/E_0.10M (left) and Amber_TIP3P_O.10M (right) simulations. The $\mathrm{K}^{+}$and $\mathrm{Cl}^{-}$ions are shown in green and cyan, respectively. For clarity, water molecules are not shown.

\footnotetext{
${ }^{1}$ Department of Medical Chemistry, Pharmaceutical Chemistry and Pharmaceutics and Bioingeeniring, University of Utah, Salt Lake City, Utah 84112

${ }^{2}$ Present address: Los Alamos National Laboratory, MS K710, Los Alamos, NM 87545
} 